\title{
Characterisation of polymorphic microsatellite loci in the freshwater bryozoan Fredericella sultana
}

\section{Journal Article}

Author(s):

Hartikainen, H.; Jokela, Jukka (D)

Publication date:

2011-06

Permanent link:

https://doi.org/10.3929/ethz-b-000044180

Rights / license:

In Copyright - Non-Commercial Use Permitted

Originally published in:

Conservation Genetics Resources 4(2), https://doi.org/10.1007/s12686-011-9578-1 


\title{
Characterisation of polymorphic microsatellite loci in the freshwater bryozoan Fredericella sultana
}

\author{
H. Hartikainen $\cdot$ J. Jokela
}

Received: 11 November 2011 / Accepted: 18 November 2011/Published online: 29 November 2011

(C) Springer Science+Business Media B.V. 2011

\begin{abstract}
Eight polymorphic microsatellite loci were isolated from massively parallel next-generation sequencing data and tested in three populations (74 individuals) of the colonial freshwater bryozoan Fredericella sultana. Up to 13 alleles per locus were found and all loci were polymorphic in all populations. Minimum of three loci were sufficient to distinguish all unique multilocus genotypes. These highly variable markers are suitable for clonal identity assignment based on unique multilocus genotypes and provide tools for resolving fine scale population structure in a species characterised by clonal, vegetative growth and asexual reproduction.
\end{abstract}

Keywords Bryozoa $\cdot$ Myxozoa $\cdot$ Proliferative kidney disease $\cdot 454 \cdot$ Microsatellite $\cdot$ Polymorphic

Freshwater bryozoans (Family Phylactolaemata, Phylum Bryozoa) are colonial suspension feeders with important ecological roles in nutrient cycling and structuring of benthic freshwater habitats (Sørensen et al. 1986). The bryozoan Fredericella sultana also acts as the primary host

H. Hartikainen · J. Jokela

Department of Aquatic Ecology, EAWAG,

Swiss Federal Institute of Aquatic Science and Technology,

Überlandstrasse 133, 8600 Dübendorf, Switzerland

H. Hartikainen · J. Jokela

ETH-Zürich, Institute of Integrative Biology,

Universitätstrasse 16, 8092 Zürich, Switzerland

Present Address:

H. Hartikainen $(\square)$

Department of Zoology, Natural History Museum,

Cromwell Road, London SW7 5BD, UK

e-mail: h.hartikainen@nhm.ac.uk for the myxozoan parasite Tetracapsuloides bryosalmonae, which causes the devastating proliferative kidney disease (PKD) of salmonid fish (Anderson et al. 1999). PKD affects many endangered salmon and trout species (Hedrick et al. 1993) and F. sultana has a key role in the persistence and spread of the PKD parasite (Okamura et al. 2011). Thus, understanding the population dynamics of $F$. sultana is crucial for explaining the recent emergence of PKD and may contribute to salmonid conservation and management.

Fredericella sultana colonies grow and spread by budding new colony modules and can reach high densities in suitable conditions. Colonies produce asexual resting stages and may disperse via colony fragments. In contrast, sexual reproduction is short in duration and may be occasionally forgone (Wood 1973). Assignment of clonal identities within such potentially homogeneous populations can be conducted using unique multilocus genotypes (MLGs), but requires highly variable markers for confident assignment. We used newly developed (e.g. Castoe et al. 2010), rapid methods utilising massively parallel 454 sequencing technology to characterize hypervariable microsatellite loci in $F$. sultana for clone identification purposes.

About $3 \mu \mathrm{g}$ of genomic DNA was isolated from whole colonies using a modified CTAB method and sequenced on a 454 Genome Sequencer FLX with Titanium chemistry at $1 / 4$ plate scale. This yielded 196,025 fragments of average 388 bp in length. The program QDD2 (Meglécz et al. 2010) was used to identify microsatellite loci from the unassembled reads and primers were designed using PRIMER 3 (as implemented in QDD2 using default settings). 936 candidate loci were identified (25\% dinucleotide loci, max. 26 repeats, $73 \%$ trinucleotide loci, max. 18 repeats and $0.1 \%$ tetranucleotide loci, max. repeats 6 ). To achieve hypervariable markers, 30 high repeat candidate loci were 
tested in 15 individuals from 5 different populations using the Qiagen Type-It microsatellite PCR kit in $8 \mu$ l reaction volumes. PCR reaction conditions were: 15 min denaturation at $95^{\circ} \mathrm{C}, 30$ cycles of $95^{\circ} \mathrm{C}(30 \mathrm{~s}), 58^{\circ} \mathrm{C}(90 \mathrm{~s})$, and $72^{\circ} \mathrm{C}(60 \mathrm{~s})$, with final elongation step at $72^{\circ} \mathrm{C}$ for $30 \mathrm{~min}$. 16 markers amplified consistently and showed putative polymorphisms when visualised on $3 \%$ agarose gels stained with ethidium bromide. Since the genomic DNA used for 454 sequencing originated from whole colonies (including guts with potential contaminating microorganisms), amplification specificity was tested using a pure $F$. sultana DNA sample as a template (obtained from resting stages only). All 16 markers amplified successfully and based on complementary fragment sizes, 8 loci were combined into one multiplex PCR using the same reagents and reaction conditions as above. The forward primers were labeled with four different fluorescent dyes (Table 1) and "pigtail" sequences (GTTTCTT) (Brownstein et al. 1996) were added to each reverse primer to reduce plus-A artefacts.

Fragment analysis of 74 individuals originating from three rivers in Switzerland was conducted using ABI PRISM 3730xl analyzer and allele sizes scored using GENEMAPPER 4.0. All loci were polymorphic and contained up to 13 alleles per locus. Locus Fs.14 failed to amplify consistently in the multiplex PCR and was removed from further analysis.
To evaluate the power of the marker set to detect unique MLGs, a test of all locus combinations was conducted in GenClone v.2.0 (Arnaud-Haond and Belkhir 2007). Minimum of three loci were sufficient to separate all unique MLGs (Fs.1, Fs.9 and Fs.13). The probabilities $\left(P_{\text {sex }}\right)$ that any pair of identical MLGs originated from distinct events of sexual reproduction were determined in GenClone (1,000 iterations). $P_{\text {sex }}$ was $<0.001$ in populations 1 and 2, suggesting that the marker set is suitable for identifying clonal genotypes with confidence. Population 3 consisted of two unique MLGs, with the dominant MLG representing $70 \%$ of all individuals. Comparisons within this population gave lower statistical confidence for excluding the possibility that some of the identical MLGs had arisen from independent events of sexual reproduction $\left(P_{\text {sex }}=0.115\right)$. In such highly clonal populations increasing the sampling effort is recommended to increase the number of distinct MLGs or further variable loci should be incorporated (Arnaud-Haond et al. 2007). Clonal heterogeneity (Simpson complement, $D^{*}$ ) based on unique MLGs was calculated in GenClone. Population 1 consisted of a diverse set of unique MLGs, with only three MLGs encountered twice each $\left(D^{*}=0.99\right)$. Fewer unique MLGs and thus higher proportion of clones were found in populations 2 and $3\left(\mathrm{D}^{*}=0.91\right.$ and $\mathrm{D}^{*}=0.44$, respectively).

Repeated MLGs were removed, which reduced the sample sizes of populations 1, 2 and 3 to 25, 16 and 2,

Table 1 Primer characteristics, amplification conditions and basic results

\begin{tabular}{|c|c|c|c|c|c|c|c|c|c|}
\hline Name & Primer sequence $\left(5^{\prime}-3^{\prime}\right)$ & $\begin{array}{l}\text { Repeat } \\
\text { type }\end{array}$ & $\begin{array}{l}\text { Primer } \\
(\mu \mathrm{M})^{\mathrm{a}}\end{array}$ & Dye & Size range ${ }^{\mathrm{b}}$ & A & $\mathrm{N}_{(1 / 2 / 3)}^{\mathrm{c}}$ & $\mathrm{H}_{0(1 / 2 / 3)}$ & $\mathrm{H}_{\mathrm{e}(1 / 2)}^{\mathrm{d}}$ \\
\hline Fs.01 & $\begin{array}{l}\text { F:TGCACCTAATGGCATGTGTT } \\
\text { R:*GTGTATGTGCACACGCTCG }\end{array}$ & $\mathrm{TG}_{22}$ & 0.6 & ATTO565 & 103-141 & $10 / 5 / 2$ & $27 / 24 / 20$ & $0.60 / \mathbf{0 . 4 4} / 0.00$ & $0.86 / 0.71$ \\
\hline Fs.04 & $\begin{array}{l}\text { F:CTCGCAGCTAATGTGGAACA } \\
\text { R:*CGCAAGAGAATGTCAGACCA }\end{array}$ & $\mathrm{GT}_{18}$ & 0.4 & YakimaYellow & $168-196$ & $8 / 5 / 3$ & $28 / 23 / 20$ & $0.64 / 0.81 / 1.0$ & $0.70 / 0.70$ \\
\hline Fs.09 & $\begin{array}{l}\text { F:CAACACATATGCGCACACAA } \\
\text { R:*GCGAGACAGATGTGCAGGTA }\end{array}$ & $\mathrm{CA}_{18}$ & 0.2 & FAM & $84-98$ & $6 / 7 / 2$ & $28 / 24 / 20$ & $0.80 / \mathbf{0 . 3 1} / 0.50$ & $0.77 / 0.74$ \\
\hline Fs. 10 & $\begin{array}{l}\text { F:CATATCTGCATTCCAGCACG } \\
\text { R:*GAATATGCTGCTGGCCGAT }\end{array}$ & $\mathrm{AC}_{18}$ & 0.2 & ATTO550 & $95-129$ & $9 / 7 / 2$ & $28 / 24 / 20$ & $0.64 / 0.94 / 0.50$ & $0.65 / 0.80$ \\
\hline Fs. 13 & $\begin{array}{l}\text { F:GAGTGATGAGAGAGTGTCGGC } \\
\text { R:*CATCCGAACAACGCAGACTA }\end{array}$ & $\mathrm{GT}_{16}$ & 0.4 & FAM & $131-157$ & $6 / 6 / 2$ & $28 / 24 / 20$ & $0.80 / 0.75 / 0.50$ & $0.78 / 0.84$ \\
\hline Fs. 14 & $\begin{array}{l}\text { F:CAGGTTCCGCTATACGTCG } \\
\text { R:*TCTTCCCCTGAAGAACGCTA }\end{array}$ & $\mathrm{GCA}_{15}$ & 0.4 & ATTO565 & na & na & na & na & na \\
\hline Fs. 18 & $\begin{array}{l}\text { F:TCTCGAAGGCTACGTTCCAC } \\
\text { R:*GTTTGACCTTACCGCGCTC }\end{array}$ & $\mathrm{TC}_{15}$ & 0.4 & ATTO550 & 136-162 & $4 / 6 / 2$ & $28 / 24 / 20$ & $0.68 / \mathbf{0 . 4 4} / 0.50$ & $0.57 / 0.70$ \\
\hline Fs. 27 & $\begin{array}{l}\text { F:TTCAAACCATTACTAGCAGCCA } \\
\text { R:*CTCCATGTGACATCCGCTTA }\end{array}$ & GTAT $_{13}$ & 0.2 & YakimaYellow & $89-121$ & $4 / 3 / 2$ & $28 / 24 / 20$ & $0.64 / \mathbf{0 . 1 3} / 0.50$ & $0.69 / 0.41$ \\
\hline
\end{tabular}

A, number of alleles; N, number of successful amplifications when sample sizes were $28 / 26 / 20$ for populations $1 / 2 / 3$; $H_{0}$ and $H_{e}$, observed and expected heterozygosity, respectively. Values in bold indicate significant departures from Hardy-Weinberg equilibrium at $P<0.05$

* Indicates a pigtail (see text)

a Concentration in multiplex PCR, ${ }^{b}$ including primer and flanking region, ${ }^{c} 1 / 2 / 3$ indicates results for each population tested, ${ }^{\mathrm{d}}$ only two unique MLGs were present in population 3 and no $\mathrm{H}_{\mathrm{e}}$ was calculated 
respectively. Linkage disequilibrium (LD) between all pairs of loci was estimated and observed and expected heterozygosities calculated in ARLEQUIN v.3.5.1.2 (Table 1). Populations 1 and 2 showed several loci with significant LD (up to five linked locus pairs in population 2). Significant heterozygote deficiency was detected in several loci within population 2 only (Table 1). Such results are not surprising given the widespread asexual reproduction and potential inbreeding within $F$. sultana populations, however, possibility of some null alleles in population 2 cannot be excluded. These first microsatellite markers for $F$. sultana will enable clone identity assignment at the population level, providing a high degree of confidence in distinguishing unique multilocus lineages.

\section{References}

Anderson CL, Canning EU, Okamura B (1999) Molecular data implicate bryozoans as hosts for PKX (Phylum Myxozoa) and identify a clade of bryozoan parasites within the Myxozoa. Parasitology 119:555-561

Arnaud-Haond S, Belkhir K (2007) GENCLONE: a computer program to analyse genotypic data, test for clonality and describe spatial clonal organization. Mol Ecol Notes 7:15-17
Arnaud-Haond S, Duarte CM, Alberto F, Serrao EA (2007) Standardising methods to address clonality in population studies. Mol Ecol 16:5115-5139

Brownstein MJ, Carpten JD, Smith JR (1996) Modulation of nontemplated nucleotide addition by Taq DNA polymerase: primer modifications that facilitate genotyping. BioTechniques 20:1004

Castoe TA, Poole AW, Gu W, de Jason Koning AP, Daza JM, Smith EN, Pollock DD (2010) Rapid identification of thousands of copperhead snake (Agkistrodon contortrix) microsatellite loci from modest amounts of 454 shotgun genome sequence. Mol Ecol Resour 10:341-347. doi:10.1111/j.1755-0998.2009.02750.x

Hedrick RP, MacConnell E, de Kinkelin P (1993) Proliferative kidney disease of salmonid fish. Annu Rev Fish Dis 3:277-290

Meglécz E, Costedoat C, Dubut V, Gilles A, Malausa T, Pech N, Martin J-F (2010) QDD: a user-friendly program to select microsatellite markers and design primers from large sequencing projects. Bioinformatics 26:403-404. doi:10.1093/bioinformatics/btp670

Okamura B, Hartikainen H, Schmidt-Posthaus H, Wahli T (2011) Proliferative kidney disease as an emerging disease: the importance of life cycle complexity and environmental change. Freshw Biol 56:735-753

Sørensen JP, Riber HH, Kowalczewski A (1986) Soluble reactive phosphorus release from bryozoan dominated periphyton. Hydrobiologia 132:145-148

Wood TS (1973) Colony development in species of Plumatella and Fredericella (Ectoprocta: Phylactolaemata). In: Boardman RS, Cheetham AH, Oliver WAJ (eds) Animal colonies development and function through time. Dowden, Hutchinson and Ross, Stroudsburg, pp 395-432 